Supporting Information for

\title{
Fabrication of Bio-based Polyelectrolyte Capsules and Their Application for Glucose-Triggered Insulin Delivery
}

\author{
Dongjian Shi ${ }^{a^{*}}$, Maoshuang Ran ${ }^{a}$, Li Zhang $^{a}$, He Huang ${ }^{a}$, Xiaojie Li $^{a}$, Mingqing \\ Chen $^{a^{*}}$, Mitsuru Akashi ${ }^{b}$
}

${ }^{a}$ The Key Laboratory of Food Colloids and Biotechnology Ministry of Education, School of Chemical and Material Engineering, Jiangnan University, Wuxi 214122, P.

R. China

b Department of Applied Chemistry, Graduate School of Engineering, Osaka University, 2-1 Yamadaoka, Suita 565-0871, Japan

Author to whom correspondence should be addressed;

E-Mail: mqchen@jiangnan.edu.cn,djshi@jiangnan.edu.cn

Tel.: +86-510-85917019; Fax: +81-510-85917763. 
FTIR spectra of chitosan (Figure S1a) and Galactose modified chitosan oligosaccharide (GC) (Figure S1b) were shown in Figure S1. Characteristic absorptions bands $\left(\mathrm{cm}^{-1}\right)$ at $3264(\mathrm{~N}-\mathrm{H}$ stretch), 2883 (C-H stretch), 1633 (amide I band), 1572 (amide II band), 1151 (C-O-C anti-symmetric stretch), and 1031 (C-O stretch) were found to assigned to the chitosan oligosaccharide. After introducing LA, all peaks of amides I and II of GC shifted from $1633 \mathrm{~cm}^{-1}$ and $1572 \mathrm{~cm}^{-1}$ to $1615 \mathrm{~cm}^{-1}$ and $1516 \mathrm{~cm}^{-1}$, respectively. Moreover, the N-H stretching peak of GC appeared at around $3264 \mathrm{~cm}^{-1}$ became slightly weaken, indicating that the amine groups of chitosan oligosaccharide and carboxyl groups of LA reacted to amide bond. FTIR results confirmed that LA successfully grafted into the CS chains.

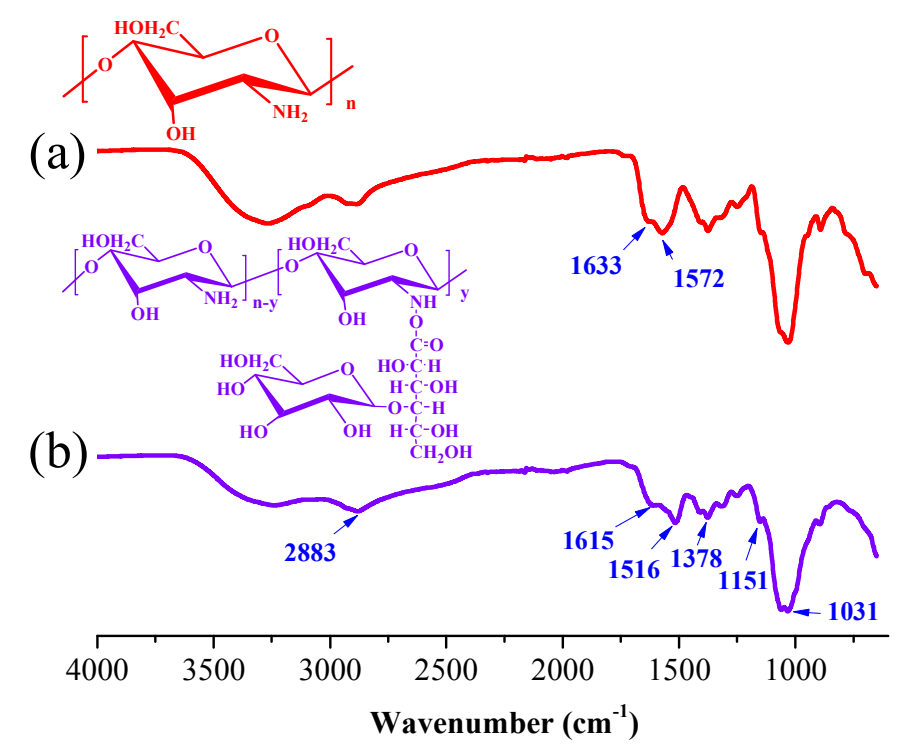

Figure S1. FTIR spectra of chitosan oligosaccharide (a) and galactosed chitosan oligosaccharide (b).

Figure S2 shows the FTIR spectra of APBA (Figure S2a), $\gamma$-PGA (Figure S2b) and $\gamma$-PGA- $g$-APBA (Figure S2c-e). The characteristic absorptions bands $\left(\mathrm{cm}^{-1}\right)$ of $\gamma$-PGA 
was as follows: 3400 (O-H stretch), $2998(\mathrm{C}-\mathrm{H}$ stretch), $1726(\mathrm{C}=\mathrm{O}$ stretch), 1532 (N-H plane bend), 1215 (C-N stretch), and 765 (N-H out-of-plane bend). From FTIR spectrum of $\gamma$-PGA-g-APBA (Figure S2c-e), three new peaks had been found at 1640 $\mathrm{cm}^{-1}, 795 \mathrm{~cm}^{-1}$ and $703 \mathrm{~cm}^{-1}$, which belonged to the amide bond and benzene ring, respectively. These results indicating APBA reacted with $\gamma$-PGA.

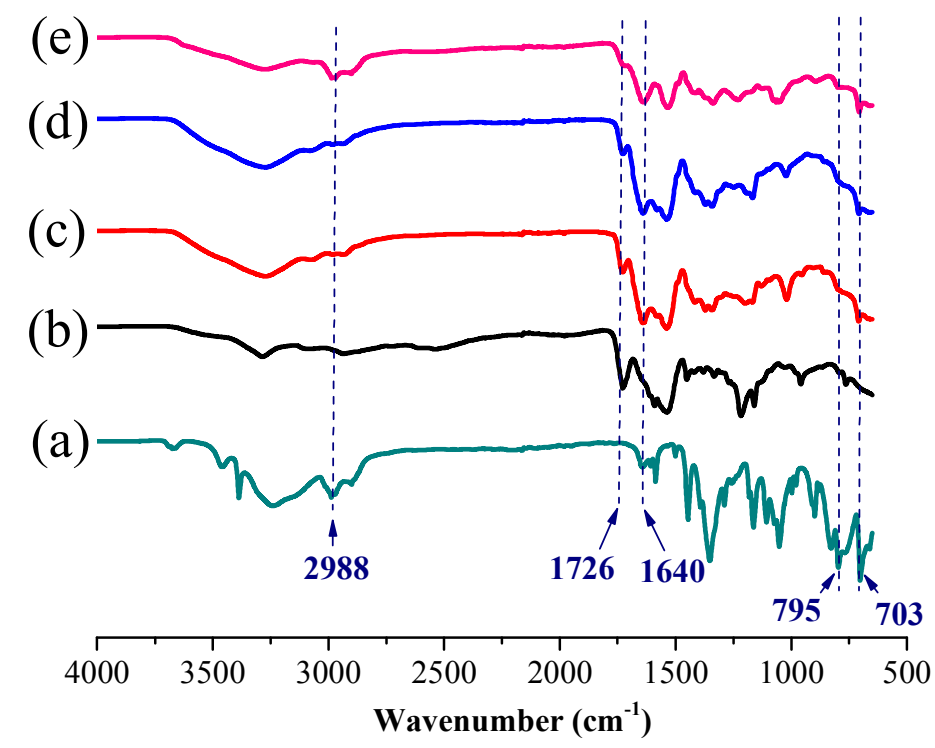

Figure S2. FTIR spectra of APBA (a), $\gamma$-PGA (b), $\gamma$-PGA- $g$-APBA 0.12 (c), $\gamma$-PGA-g-APBA 0.29 (d) and $\gamma$-PGA-g-APBA $0.40(e)$

Table S1. Synthesis and characterization of $\gamma$-PGA- $g$-APBA

\begin{tabular}{cccc}
\hline $\begin{array}{c}\text { Samples. } \\
\gamma \text {-PGA/APBA/EDC/NHS } \\
(\mathrm{mmol})\end{array}$ & $\begin{array}{c}\text { Graft ratio } \\
(\%)^{\mathrm{a}}\end{array}$ & $\begin{array}{c}\text { Zeta potential } \\
(\mathrm{mV})^{\mathrm{b}}\end{array}$ \\
\hline$\gamma$-PGA-g-APBA 0.12 & $6 / 1.5 / 1.5 / 4.5$ & $12 \%$ & $-29.51 \pm 0.15$ \\
$\gamma$-PGA-g-APBA 0.29 & $6 / 3 / 3 / 9$ & $29 \%$ & $-19.19 \pm 0.15$ \\
$\gamma$-PGA-g-APBA 0.40 & $6 / 4.5 / 4.5 / 13.5$ & $40 \%$ & $-12.35 \pm 0.15$ \\
\hline
\end{tabular}

${ }^{\mathrm{a}}$ Graft ratio was determined by ${ }^{1} \mathrm{H}-\mathrm{NMR} ;{ }^{\mathrm{b}}$ Zeta potential was obtained from Zeta PALS at $\mathrm{pH} 5$. 


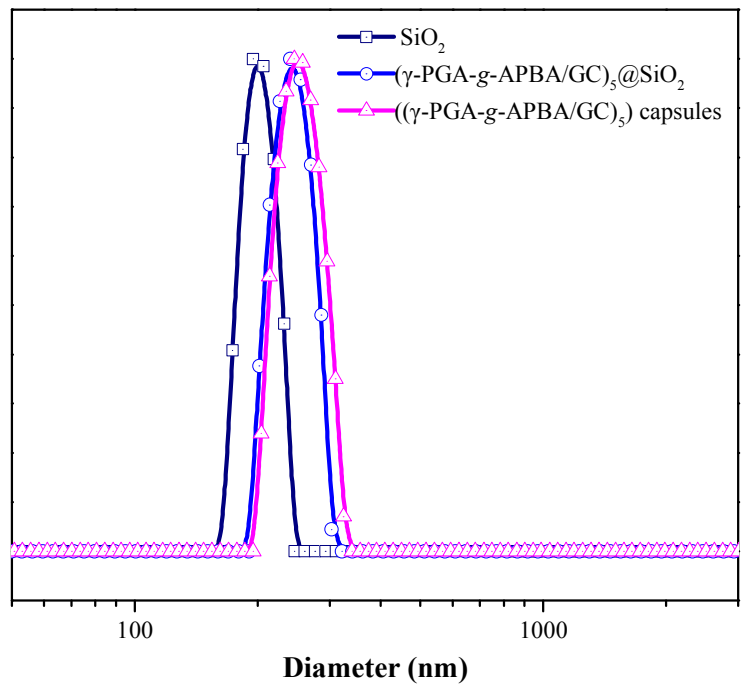

Figure S3. Dynamic light scattering (DLS) of the $\mathrm{SiO}_{2}$ nanoparticles, $(\gamma$-PGA-gAPBA/GC) $)_{5} @ \mathrm{SiO}_{2}$ nanoparticles and ( $\gamma$-PGA-g-APBA/GC) $)_{5}$ capsules.
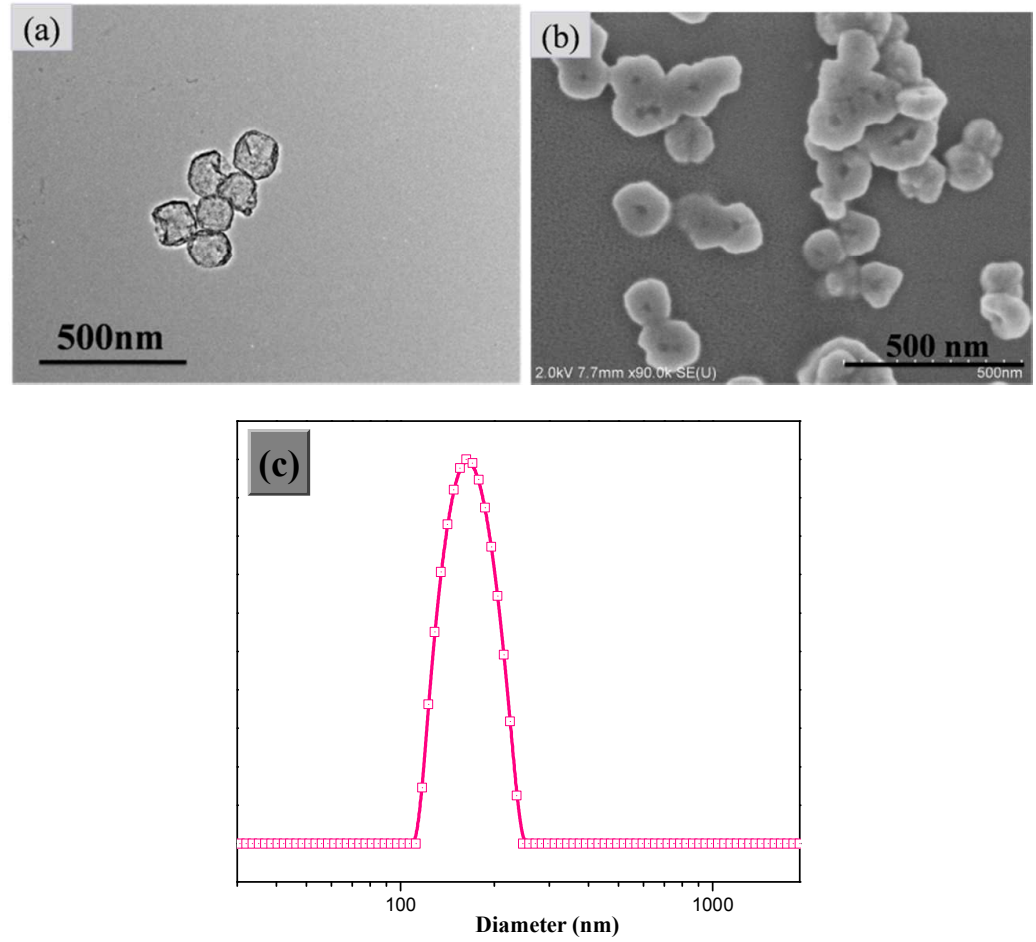

Figure S4. TEM (a) and SEM (b) images and DLS (c) curve of $(\gamma \text {-PGA- } g \text {-APBA/GC })_{5}$ capsules after freeze-drying process. 

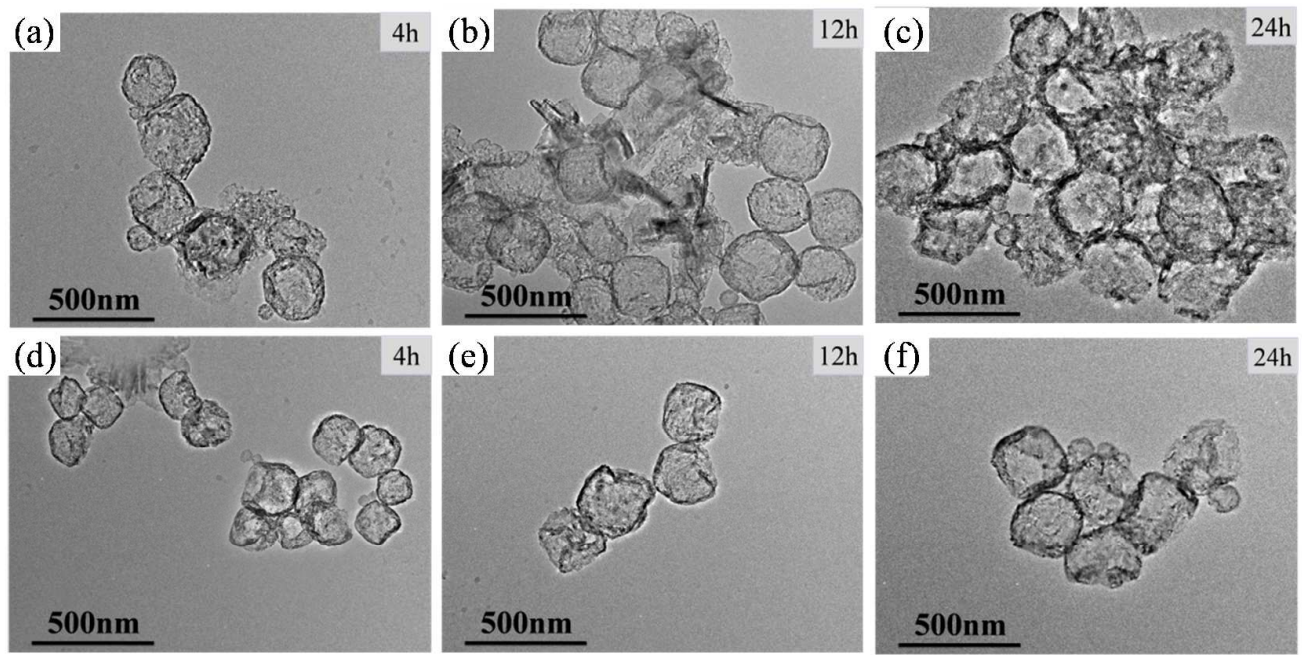

Figure S5. Morphologies of the $\left(\gamma-\mathrm{PGA}-\mathrm{g}-\mathrm{APBA}_{0.29} / \mathrm{GC}\right)_{5}(\mathrm{a}-\mathrm{c})$ and $\left(\gamma \text {-PGA-g-APBA }{ }_{0.40} / \mathrm{GC}\right)_{5}(\mathrm{~d}-\mathrm{f})$ capsules with time at $20 \mathrm{mg} / \mathrm{mL}$ of glucose solution.
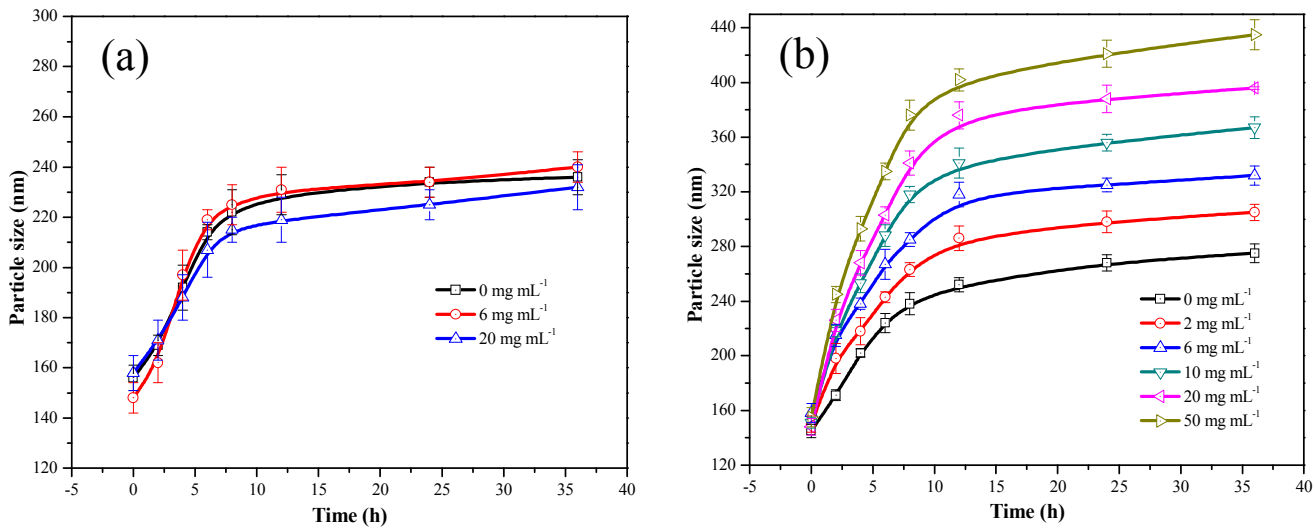

Figure S6. Glucose sensitivity of the $(\gamma \text {-PGA/CS })_{5}(\mathrm{a})$ and $\left(\gamma-\mathrm{PGA}-g-\mathrm{APBA}_{0.29} / \mathrm{CS}\right)_{5}$

(b) capsules in PBS solution ( $\mathrm{pH} 7.4$ ) with different glucose levels at $37^{\circ} \mathrm{C}$. 


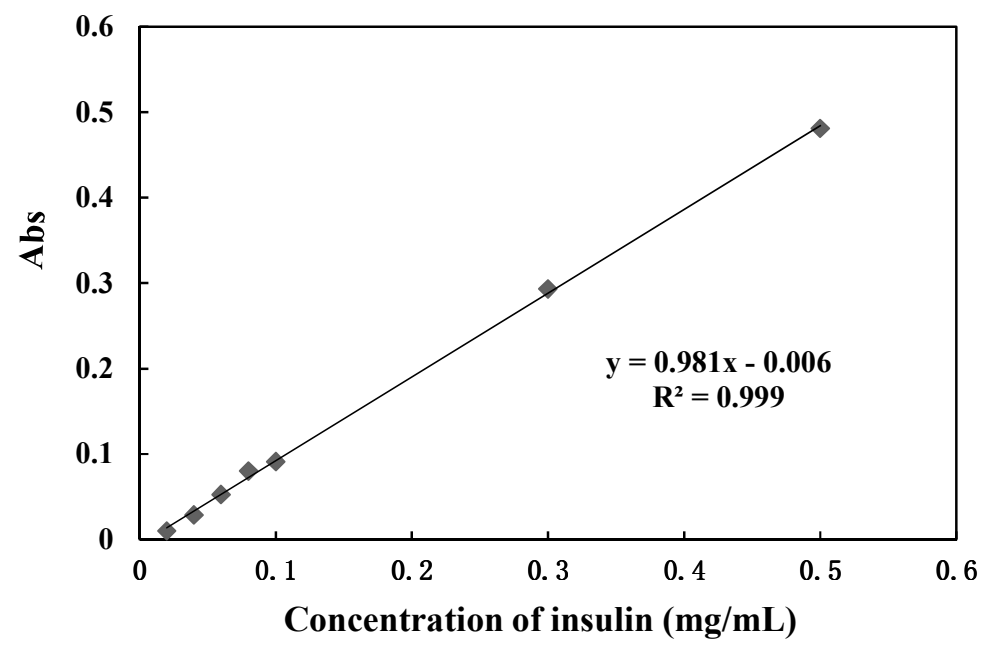

Figure S7. Insulin calibration curve generated by spectrophotometer at $595 \mathrm{~nm}$ from a series of insulin w different concentrations using coomassie brilliant blue (G-250) as visual indicator.

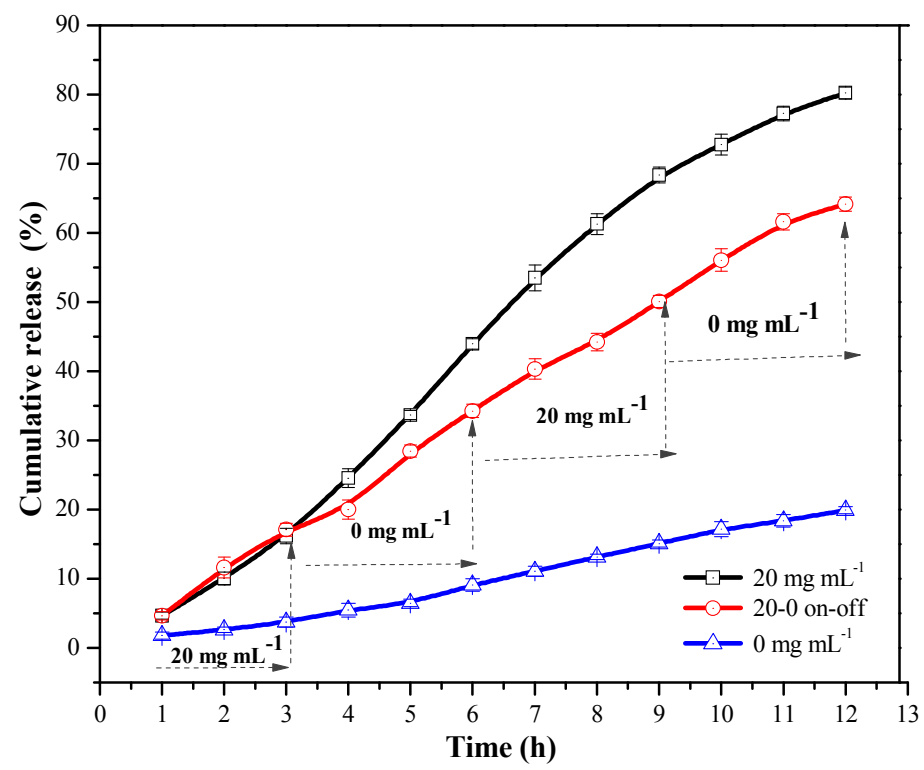

Figure S8. Insulin cumulative release from the $\left(\gamma-\mathrm{PGA}-g-\mathrm{APBA}_{0.29} / \mathrm{CS}\right)_{5}$ capsules in PBS triggered by alternant glucose concentrations at $20-0 \mathrm{mg} / \mathrm{mL}$. 


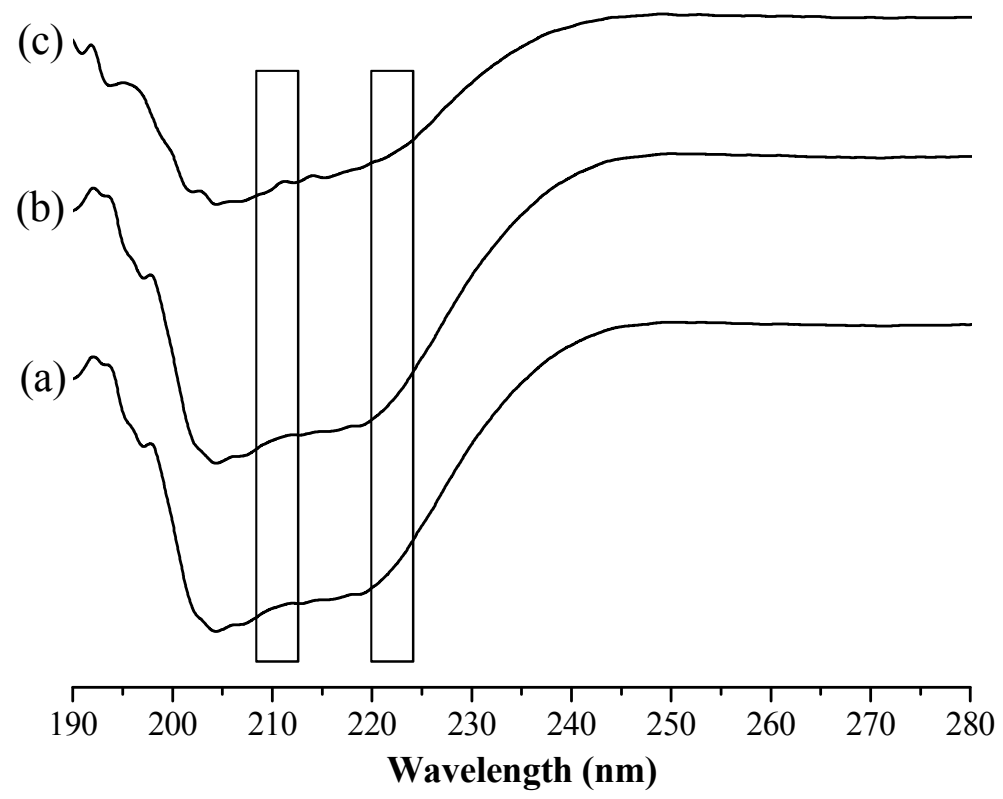

Figure S9. Circular dichroism (CD) spectra of the native insulin in PBS at $\mathrm{pH} 7.4$ (a) and at $\mathrm{pH} 5.5$ (b) and insulin released after $12 \mathrm{~h}$ from the capsules (c).
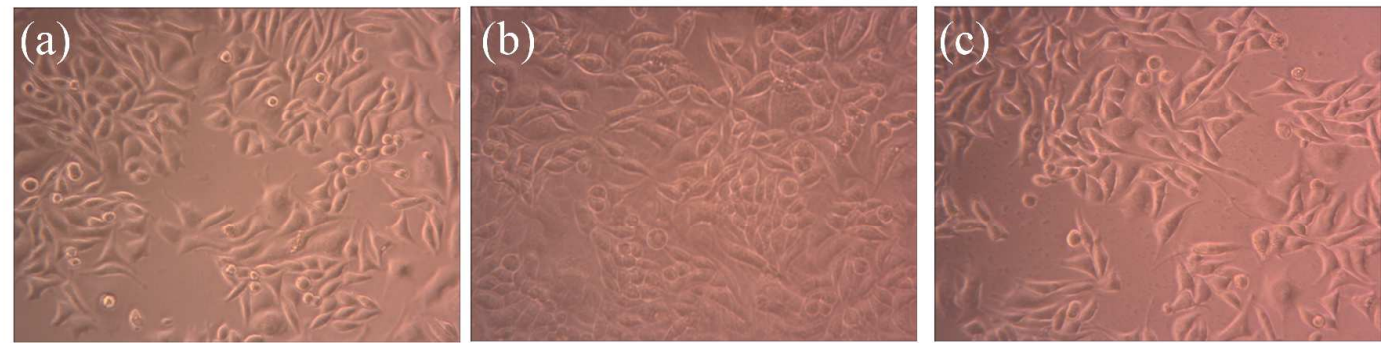

Figure S10. Inverted microscope images of the $\left(\gamma-\mathrm{PGA}-g-\mathrm{APBA}_{0.12} / \mathrm{GC}\right)_{5}(\mathrm{a})$, $\left(\gamma \text {-PGA- } g \text {-APBA }{ }_{0.29} / \mathrm{GC}\right)_{5}(\mathrm{~b})$ and $\left(\gamma \text {-PGA- } g \text {-APBA }{ }_{0.40} / \mathrm{GC}\right)_{5}(\mathrm{c})$ capsules after incubation for $24 \mathrm{~h}$ at $37^{\circ} \mathrm{C}$. 